$48 / 19192 \mathrm{gm}$

PPPL-2843 UC-427
(2)

PREPARED FOR THE U.S. DEPARTMENT OF ENERGY, UNDER CONTRACT DE-AC02-76-CHO-3073

PPPL-2843

\title{
TRAPPED PARTICLE DYNAMICS \\ IN TOROIDALLY ROTATING PLASMAS
}

BY

T.S. HAHM

June, 1992

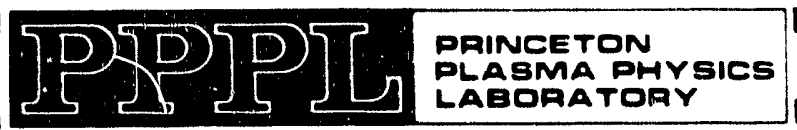

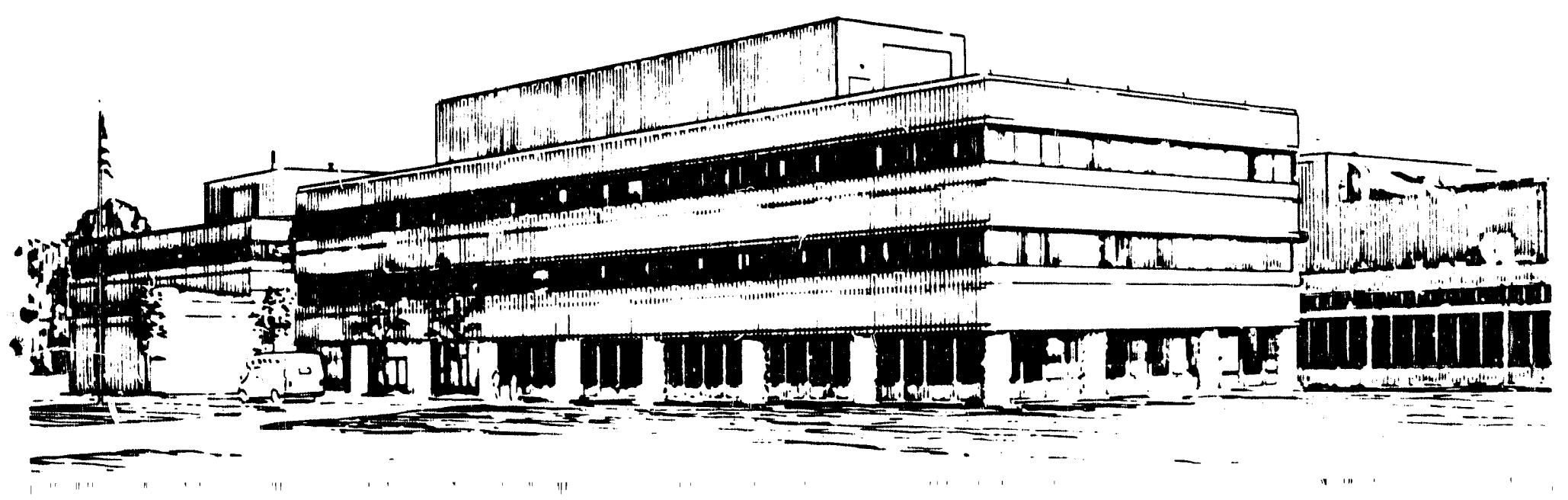




\section{NOTICE}

This report was prepared as an account of work sponsored by an agency of the United States Government. Neither the United States Government nor any agency thereof, nor any of their employees, makes any warranty, express or implied, or assumes any legal liability or responsibility for the accuracy, completeness, or usefulness of any information, apparatus, product, or process disclosed, or represents that its use would not infringe privately owned rights. Reference herein to any specific commercial produce, process, or service by trade name, trademark, manufacturer, or otherwise, does not necessarily constitute or imply its endorsement, recommendation, of favoring by the United States Government or any agency thereof. The views and opinions of authors expressed herein do not necessarily state or reflect those of the United States Government or any zency thereof.

\section{NOTICE}

This report has been reproduced directly from the best available copy.

Available to DOE and DOE contractors from the:

Office of Scientific and Technical Information

P.O. Box 62

Oak Ridge, TN 37831;

Prices available from (615) 576-8401.

Available to the public from the:

National Technical Information Service

U.S. Department of Commerce

5285 Port Royal Road

Springfield, Virginia 22161

$703-487-4650$ 


\title{
Trapped Particle Dynamics in Toroidally Rotating Plasmas
}

\section{T.S. Hahm}

\section{Princeton Plasma Physics Laboratory, Princeton University Princeton, N.J.}

\begin{abstract}
A detailed single particle orbit analysis in toroidally rotating plasma yielcis new analytical formulas for the second adiabatic invariant, the bounce frequency, and the precession frequency up to the first order correction in $\rho_{\text {pi }}$ (poloidal ion gyroradius) $/ L_{v}($ scale length of rotation velocity), for toroidal flow values of the order of ion thermal velocity. Toroidal plasma rotation effects on the trapped ion instabilities in tokamaks are investigated in the context of local theory. Toroidal plasma rotation increases both the fraction of trapped particles and their precession drift velocity. Consequently, the growth rate of trapped ion instability increases in both dissipative and collisionless regimes.
\end{abstract}

PACS numbers: $52.35 . \mathrm{Kt}, 52.20 . \mathrm{Dq}, 52.35 . \mathrm{Py}, \quad 52.55 . \mathrm{Fa}$ 


\section{Introduction}

Microturbulence in high temperature tokamak plasmas remains an important and challenging topic. In most cases, the spectral intensity of density flustuation measured by microwave scattering peaks at the longest measurable wavelengths $\left(k_{\perp} \rho_{s} \leq 0.2\right)$ within the spatial resolution. 1,2 New diagnostics on TFTR such as reflectometry ${ }^{3}$ and beam emission spectroscopy ${ }^{4}$ will address the problem of identifying the peak of $\mathrm{k}$-spectrum in the near future. Since the usual electron drift waves, such as trapped electron modes, exhibit the linear growth rate maximum at a relatively short wavelength $\left(k_{\perp} \rho_{S} \approx 1\right)$, it is crucial to study the influence of longer wavelength instabilities such as trapped ion modes on transport in tokamaks. Of course, it is premature to conclude that trapped electron modes are relatively unimportant. In fact, there exists a number of nonlinear theories which predict spectral transfer to longer wavelengths $c$.ue to ion Compton scattering. $5 \cdot 8$

High temperature, low collisionality plasmas (in which the trapped particle modes are likely to be relevant) are often produced through neutral beam heating, and rotate toroidally with significant speed (for instance $V_{O \varphi} C_{S} \geq 0.5$, in TFTR hot ion-mode ${ }^{9}$ ) for unbalanced neutral beam injection case. In this case, Doppler shifted frequency of measured density fluctuation dominates diamagnetic drift frequency, making it harder to identify the propagation direction of fluctuation in the plasma frame. Therefore, it is important to include the effects of plasma rotation in the analysis of microinstabilities. So far, plasma rotation effects on trapped particle instabilities have been analyzed with a focus on the role of radially dependent Dopper-shifted in quency in slab geometry. ${ }^{10-13}$

In this paper, we investigate the effects of plasma toroidal rotation on trapped ion instability. We focus on the effects of orbit modification in the context of local theory. ${ }^{14}$ Since a rotating frame in toroidal geometry is no longer an inertial frame, we get significant effect even for rigid toroidal rotation. Nonlocal analysis in a toroidal geometry is beyond the scope 
of this paper, and probably requires numerical studies. 15 We present a detailed orbit analysis of trapped particles in the presence of toroidal rotation. We derive the new analytical formulas for the second adiabatic invariant, the bounce frequency, and the precessional frequency up to the Iirst order correction in $\rho_{\mathrm{pi}} / \mathrm{L}_{\mathrm{v}}$, for toroidal flow values of the order of ion thermal velocity. Changes in those frequencies are expected to modify the strength of the wave-particle interaction of various resonant instabilities. Principal results of this paper are:

i) Toroidal rotation induced radial electric field enhances the magnetic trapping of ions, meanwhile the centrifugal force induced poloidal electric field enhances the magnetic trapping of electrons. This increases the fraction of trapped particles and their precession frequencies.

ii) Consequently, the growth rate of trapped ion instability increases in both dissipative and collisionless regime.

iii) The banana radial width of a trapped ion in the presence of rotation shear is derived, and a discussion is given to elucidate the relation between two seemingly different previous results. 16,17

The remainder of this paper is organized as follows. In Sec. II, a detailed single particle orbit analysis is presented in ine presence of sheared toroidal rotation. In Sec. III, the growth rates of trapped ion instabilities are calculated in both dissipative and collisionless regimes. Finally, the implications of the orbit modification results on the trapped electron instabilities are discussed in Sec. IV. 


\section{Single Particle Orbit Analysis}

We start from a trapped ion orbit analysis. We consider a toroidally rotating plasma with toroidal flow values of the order of $\mathrm{V}_{\mathrm{Ti}}\left(\mathrm{V}_{O \varphi} \leq \mathrm{V}_{\mathrm{Ti}}\right)$, and its gradient scale length much larger than the poloidal Larmor radius of ions, i.e., $L_{v}=\left(d \ln V_{o \varphi} / d l n r\right)^{-1} \gg \rho_{p i}=$ $\mathrm{cMV}_{\mathrm{Ti}_{\mathrm{i}}} / \mathrm{eB}_{\mathrm{p}}$. Focusing our interests mainly on core region of tokamaks where the poloidal plasma rotation is expected to be quickly damped, the following lowest order radial force balance is assumed. 18,19

$$
E_{r}(0)+V_{O \varphi} B_{p}=0
$$

This yields $V_{O \varphi}=\omega(\Psi) R$ with $\omega(\Psi)=-d \Phi_{O} / d \Psi$.

Using the gyrocenter (guiding center) variables 20 which include the ellipticity of Larmor orbit due to the radial dependence of $\mathrm{V}_{O \varphi}(\mathrm{r})$, the following formulas for the canonical angular momentum and the energy of a single ion gyrocenter are obtained.

$$
\begin{aligned}
& P \phi=-e \Psi+M R\left(R \omega(\Psi)+c_{\|}\right), \\
& E=M\left(c_{\|}+\omega(\Psi) R\right)^{2} / 2+\mu B+e \Phi+M V_{E}^{2 / 2},
\end{aligned}
$$

where $c_{\|}=V_{\|}-V_{o \varphi}(r)$. Here, a high aspect ratio tokamak with $\mathbf{b} \cdot \phi \equiv 1$ is considered. Therefore, the last term in Eq. (3) can be ignored up to the first order in $\varepsilon / q$. Small $\rho_{p i} / L_{v}$ makes the lowest order expression for the magnetic moment $\mu=M c_{\perp}{ }^{2} / 2 \mathrm{~B}$ [where $\mathrm{c}_{\perp}=$ $\left.V_{\perp}-V_{E}(r)\right]$ sufficient for our present purposes. 
For a trapped ion orbit analysis, it is convenient to remove $\Psi$-dependence from Eq. (3) using Eq. (2). Since $c_{\|}=0$ at the mirror points of trapped ion orbits, Eq. (2) yields

$$
\Psi-\Psi_{0}=(\mathrm{M} / \mathrm{e})\left\{\mathrm{R} \mathrm{c}_{\|}+\mathrm{R}^{2} \omega(\Psi)-\mathrm{R}(0)^{2} \omega\left(\Psi_{0}\right)\right\}
$$

Although we are primarily interested in trapped particle orbits, a similar procedure could be extended to passing particle orbit analysis by choosing $\Psi_{0}$ which satisfies $\mathrm{P}_{\phi}=-\mathrm{e} \Psi_{\mathrm{O}^{+}}$ $\mathrm{MR}_{0}{ }^{2} \omega\left(\Psi_{0}\right)$. Using $\omega(\Psi) \equiv \omega\left(\Psi_{0}\right)+\left(\Psi-\Psi_{0}\right)(\partial \omega / \partial \Psi)_{0}$ and Eq. (4), we get

$$
R^{2} \omega(\Psi)-R(0)^{2} \omega\left(\Psi_{0}\right)=S^{-1}\left\{R(0)^{2}(\partial \omega / \partial \Psi)_{0} R c_{\|}+\left(R^{2}-R(0)^{2}\right) \omega\left(\Psi_{0}\right)\right\}
$$

where $S=1-R(0)^{2}(\partial \omega / \partial \Psi)_{0}$ is the orbit modification parameter ${ }^{16}$ when Eq. (1) is satisfied. An alternative expression for Eq. (4) is

$$
\Psi-\Psi_{0}=(\mathrm{M} / \mathrm{e}) \mathrm{S}^{-1}\left\{\mathrm{Rc}_{\|}+\left(\mathrm{R}^{2}-\mathrm{R}(\mathrm{o})^{2}\right) \omega\left(\Psi_{\mathrm{O}}\right)\right\}
$$

Using $\Phi_{0}(\Psi) \equiv \Phi_{0}\left(\Psi_{0}\right)-\omega\left(\Psi_{0}\right)\left(\Psi-\Psi_{0}\right)-(\partial \omega / \partial \Psi)_{0}\left(\Psi-\Psi_{0}\right)^{2} / 2$, Eq. (4) and Eq. (6), we get

$$
E=M c_{\|}^{2} / 2 S+\mu B-M \omega\left(\Psi_{0}\right)^{2} R^{2} / 2+M \omega\left(\Psi_{0}\right)^{2} R(0)^{2}+e \Phi_{0}\left(\Psi_{0}\right)+e \Phi_{1}
$$

The third term describes the centrifugal force produced by toroidal rotation. Here, we keep the poloidal angle dependent potential $\Phi_{1}{ }^{21}$ Because of the centrifugal force, the ion density is pushed outward in major radius, yielding

$$
n_{i}=N(\Psi) \exp \left(M \omega\left(\Psi_{0}\right)^{2}\left(R^{2}-\left\langle R^{2}\right\rangle\right) / 2 T_{i}-e \Phi_{1} / T_{i}\right\}
$$


where $\left\langle R^{2}\right\rangle$ is the flux surface average of $R^{2}$. Meanwhile, the electron density remains a Boltzman response along the magnetic field line due to negligible electron mass,

$$
n_{e}=N(\Psi) \exp \left(e \Phi_{1} / T_{e}\right)
$$

Therefore, a poloidal electrostatic field is produced to maintain charge neutrality. From Eqs. (8) and (9), we get $^{18,19}$

$$
e \Phi_{1}=M \omega\left(\Psi_{0}\right)^{2}\left(R^{2}-\left\langle R^{2}\right\rangle\right) T_{e} / 2\left(T_{i}+T_{e}\right)
$$

After a high aspect ratio expansion, Eq. (7) becomes

$$
\begin{aligned}
E= & M c_{\|}{ }^{2} / 2 S+\mu B_{0}(1-\varepsilon \cos \Theta)+M \omega\left(\Psi_{0}\right)^{2} R(0)^{2}-M \omega\left(\Psi_{0}\right)^{2} R_{0}^{2 / 2}+e \Phi_{0}\left(\Psi_{0}\right) \\
& -M \omega\left(\Psi_{0}\right)^{2} R_{0}{ }^{2} T_{i}\left(T_{i}+T_{e}\right)^{-1} \varepsilon \cos \Theta .
\end{aligned}
$$

It is interesting to note that the third term in Eq. (7) which is responsible for the centrifugal force, is partially cancelled by $\mathrm{e} \Phi_{1}$. As a result, the last term in Eq. (11) modifies the effective potential for the single ion motion in $\Theta$-direction. Eq. (11) can be written as

$$
c_{\|}^{2}=4 \varepsilon S M^{-1}\left\{\mu B_{0}+M \omega\left(\Psi_{0}\right)^{2} R_{0}{ }^{2} T_{i}\left(T_{i}+T_{e}\right)^{-1}\right\}\left\{\kappa^{2}-\sin ^{2} \Theta / 2\right\}
$$

where $E_{e f f}=E-M \omega\left(\Psi_{0}\right)^{2} R(0)^{2}+M \omega\left(\Psi_{0}\right)^{2} R_{0}^{2 / 2}-e \Phi_{0}\left(\Psi_{0}\right)$, and

$$
\kappa^{2}=1 / 2+\left(E_{\text {eff }}-\mu B_{0}\right) / 2 \varepsilon\left\{\mu B_{0}+M \omega\left(\Psi_{0}\right)^{2} R_{0}^{2} T_{i}\left(T_{i}+T_{e}\right)^{-1}\right\}
$$


In Eq. (13), $x$ denotes the pitch angle of trapped ions, i.e., $\Theta_{0}=2 \sin ^{-1} K$ is the poloidal location of mirror point. Deeply trapped particles correspond to $k \ll 1$, meanwhile barely trapped particles have $\alpha$ values less than, but near 1 .

Now, we calculate the second adiabatic invariant, $J=(2 \pi)^{-1} \int_{P_{\Phi}} \phi \cdot b d l$. Using Eq. (2) and performing standard manipulations involving elliptic integrals, we get

$$
J=2 q R_{0} \pi^{-1}\left[\varepsilon S M^{-1}\left(\mu B_{0}+M \omega\left(\Psi_{0}\right)^{2} R_{o}^{2} T_{i}\left(T_{i}+T_{e}\right)^{-1}\right\}\right]^{1 / 2}\left\{\left(\kappa^{2}-1\right) K(\kappa)+E(\kappa)\right\}
$$

where $K(\kappa)$ and $E(\kappa)$ are complete elliptic integrals of the first and second kind, respectively. The bounce frequency of a trapped ion is

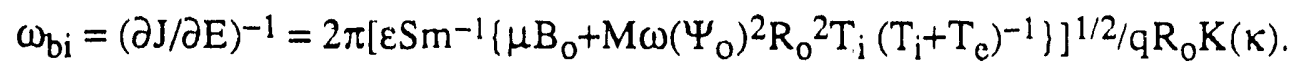

A similar formula has been independently obtained in the absence of poloidal electric field. ${ }^{17}$ Also, a limiting case of deeply trapped particle in the absence of poloidal electric field and velocity shear has been previously considered in Ref.22, and can be recovered by taking $\kappa \rightarrow 0$ and $S \rightarrow 1$ limit of Eq.(15). Equation (15) also shows the trends observed in the numerical studies of test particles in Ref.23 for a rigid rotation case $(S=1), \omega_{\text {bi }}$ increases when $\omega\left(\Psi_{0}\right)$ is raised with $\mathrm{k}$ fixed or with $c_{\|}(\theta=0)$ fixed. The toroidal precession drift frequency is given by

$$
\omega_{d i}=k_{\Phi} R_{0} \omega_{b i}(\partial J / \partial \Psi)=-\left\{\mu B_{0}+M \omega\left(\Psi_{0}\right)^{2} R_{0} 2 T_{i}\left(T_{i}+T_{e}\right)^{-1}\right\} 2 c n q G(\kappa) / e B_{i} n
$$

where $G(\kappa)=4 s\left\{\left(\kappa^{2}-1\right) K(\kappa)+E(\kappa)\right\}+2 E(\kappa)-K(\kappa)$. This formula has been previously derived only in the absence of plasma rotation. ${ }^{14}$ This new result implies interesting consequences for the trmpped ion instability which we discuss in the next section. We note that $\left|\omega_{\mathrm{di}}\right|$ increases when $\omega\left(\Psi_{0}\right)$ is raised with $\mathrm{x}$ fixed. Inspection of Eq. (12) shows that trapped 
ions should satisfy

$$
c_{\|}(\theta=0)^{2} \leq 4 \varepsilon S M^{-1}\left\{\mu B_{0}+m \omega\left(\Psi_{0}\right)^{2} R_{0}^{2} T_{i}\left(T_{i}+T_{e}\right)^{-1}\right\}
$$

Equation (17) also defines the boundary between the trapped and untrapped particles in $\left(c_{\|}(\theta=0),\left(\mu B_{0}\right)^{1 / 2} / M\right)$ space. This curve has a hyperbolic shape with an asymptotic slope given by $\left(\mu \mathrm{B}_{0}\right)^{1 / 2} / \mathrm{Mc}_{\|}(\Theta=0)=(4 \varepsilon S)^{-1 / 2}$. We note that low energy particles with $c_{\|}(\theta=0)^{2} \leq 4 \varepsilon S$ $\omega\left(\Psi_{0}\right)^{2} R_{0}^{2} T_{i}\left(T_{i}+T_{e}\right)^{-1}$ are electrostatically trapped regardless of $\mu$ values.

Now, we calculate the trapped electron orbit modification. Since an electron has negligible mass, its radial excursion from $\Psi_{\mathrm{O}}$ is smaller than that of trapped ion by $(\mathrm{m} / \mathrm{M})^{1 / 2}$. Therefore, we can ignore the $\Psi$ dependence of $\Phi_{0}(\Psi)$. However, a poloidal electric field due to $\Phi_{1}$ has significant effects on trapped electron orbits. The single electron energy is

$$
\begin{aligned}
& E=m c_{\|}{ }^{2} / 2+\mu_{e} B-e \Phi_{0}(\Psi)-e \Phi_{1} \\
& =m c_{\|}{ }^{2} / 2+\mu_{e} B_{0}(1-\varepsilon \cos \Theta)-e \Phi_{0}\left(\Psi_{0}\right)-M \omega\left(\Psi_{0}\right)^{2} R_{0}{ }^{2} T_{e}\left(T_{i}+T_{e}\right)^{-1} \varepsilon \cos \Theta,
\end{aligned}
$$

which leads to

$$
c_{\|}^{2}=4 \varepsilon m^{-1}\left\{\mu_{\mathrm{e}} \mathrm{B}_{\mathrm{o}}+\mathrm{M} \omega\left(\Psi_{\mathrm{o}}\right)^{2} \mathrm{R}_{\mathrm{o}}^{2} \mathrm{~T}_{\mathrm{e}}\left(\mathrm{T}_{\mathrm{i}}+\mathrm{T}_{\mathrm{e}}\right)^{-1}\right\}\left\{\kappa^{2}-\sin ^{2} \Theta / 2\right\}
$$

where $E_{\text {eff }}=E+e \Phi_{0}\left(\Psi_{0}\right)$, and

$$
\kappa^{2}=1 / 2+\left(E_{e f f}-\mu_{e} B_{0}\right) / 2 \varepsilon\left(\mu_{e} B_{o}+M \omega\left(\Psi_{0}\right)^{2} R_{0}^{2} T_{e}\left(T_{i}+T_{e}\right)^{-1}\right)
$$

Now, we can also calculate the following formulas for the trapped electrons using similar methoci. The longitudinal (second) adiabatic invariant $J=(2 \pi)^{-1} \int_{P_{\Phi}} \phi \cdot b d l$ is 


$$
J=2 q R_{0} \pi^{-1}\left[\varepsilon m^{-1}\left(\mu_{e} B_{o}+M \omega\left(\Psi_{0}\right)^{2} R_{o}^{2} T_{e}\left(T_{i}+T_{e}\right)^{-1}\right\}\right]^{1 / 2}\left\{\left(\kappa^{2}-1\right) K(\kappa)+E(\kappa)\right\},
$$

The bounce frequency of a trapped electron is

$$
\omega_{b e}=(\partial J / \partial E)^{-1}=2 \pi\left[\varepsilon m^{-1}\left(\mu_{e} B_{o}+M \omega\left(\Psi_{o}\right)^{2} R_{o}^{2} T_{e}\left(T_{i}+T_{e}\right)^{-1}\right)\right]^{1 / 2 / q R_{o} K(\kappa)}
$$

The toroidal precession drift frequency is given by

$\omega_{\text {de }}=k_{\Phi} R_{0} \omega_{b e}(\partial J / \partial \Psi)=\left(\mu_{e} B_{0}+M \omega\left(\Psi_{0}\right)^{2} R_{0}^{2} T_{e}\left(T_{i}+T_{e}\right)^{-1}\right\} 2 c n q G(x) / e B_{0} R_{0}$

where $G(x)=4 s\left\{\left(x^{2}-1\right) K(\kappa)+E(\kappa)\right\}+2 E(x)-K(\kappa)$. This new result implies interesting consequences on the collisionless trapped electron modes as well as the trapped ion modes as will be discussed later.

Finally, we discuss the radial width of bananas 24,25 in the presence of sheared toroidal rotation. This issue has been discussed extensively in relation to the edge sheath 16 and $\mathrm{L}$ to $\mathrm{H}$ transition physics. 17,22 The full radial width can be easily obtained from Eq. (6),

$$
\rho_{\mathrm{bi}}=\Delta \Psi / \mathrm{RB}_{\mathrm{p}}=2 \mathrm{Mc} \mathrm{c}_{\|}(\Theta=0) / \mathrm{eB}_{\mathrm{p}} \mathrm{S}
$$

We note that a limiting case of this formula has been previously obtained for the rigid rotation case $(S=1), 26,23$ Equation (24) indeed shows the trends obtained by test particle numerical studies in Ref. 23, i.e, $\rho_{b i}$ increases when $V_{o \varphi}$ is raised with $\kappa$ fixed, meanwhile $\rho_{b i}$ does not change much when $V_{O \varphi}$ is raised with $c_{\|}(\Theta=0)$ fixed. In the presence of velocity shear, a similar formula has been previously obtained in terms of different variables. ${ }^{17}$ In that work, the inverse proportionality of $\mathrm{P}_{\mathrm{bi}}$ to the orbit modification factor $\mathrm{S}$ has been emphasized. At a first glance, this does not appear to agree with the results in Ref. 16 which indicisie that the banana 
radial width is reduced by $S^{1 / 2}$. Here, we discuss their relations. We emphasize that our analysis assumes $L_{v} \gg \rho_{p i}$, and is valid when $|S-1| \ll 1$, while the results in Ref. 16 and Ref. 17 are intended for application to the plasma edge shear layer $27-30$ where $S$ values encountered could be significantly different from 1 . When test particles are injected into a plasma with given $V_{o \varphi}$, Eq. (24) is useful and $S$ is the appropriate orbit squeezing factor. However, if we compare "typical" barely trapped ions, the appropriate orbit squeezing factor should be $S^{1 / 2}$ as stated in Ref. 16. This can be shown by noticing the proportionality of $c_{\|}(\theta=0)$ to $S^{1 / 2}$ in Eq. (17). In the limit of small $V_{o \varphi}$ but finite $|S-1|$, we get $c_{\|}(\theta=0) \approx$ $(\mathrm{SE})^{1 / 2} \mathrm{~V}_{\mathrm{Ti}}$ which leads to

$$
\rho_{\mathrm{bi}} \approx 2 \mathrm{M} \varepsilon^{1 / 2} \mathrm{~V}_{\mathrm{Ti}} / \mathrm{eB} \mathrm{B}_{\mathrm{p}} \mathrm{S}^{1 / 2}=\rho_{\mathrm{bi}}\left({ }^{(0) / \mathrm{S}^{1 / 2}}\right.
$$

where $\rho_{b i}{ }^{(0)}$ is the typical banana width in the absence of velocity shear. 


\section{Trapped Ion Instability in The Presence of Toroidal Rotation}

In this section, we investigate the trapped particle orbit modification effects on trapped ion instability. For simplicity, long wavelength modes $\left(k_{\perp} \rho_{b i}<<1\right)$ are considered for the rigid rotation case $(S=1)$ in the local limit. First, we calculate the fraction of trapped ion population. For the sake of comparison, we take a local Maxwellian distribution function in a toroidally moving frame, i.e., $F_{0} \propto \exp \left(-E T_{i}\right)$. Then the fraction $\delta$ at $\Psi_{0}$ and $\theta=0$ is given by

$\delta=\int_{T r} d \mu \int_{d c_{\|}} \exp \left\{-M c_{\|}{ }^{2} / 2-\mu B_{0}(1-\varepsilon)\right\} / \int_{d \mu} \iint_{d c_{\|}} \exp \left\{-M c_{\|}{ }^{2} / 2-\mu B_{v}(1-\varepsilon)\right\}$,

where the integration variables are appropriately normalized to $\mathrm{V}_{\mathrm{Ti}}$. Although $\mathrm{Eq}$. (26) can be expressed in terms o! Error function, we can obtain simple asymptotic formulas by changing the order of integration. For $\varepsilon \ll 1$, the asymptotic behavior of $\delta$ is

$$
\delta=(2 \varepsilon)^{1 / 2} \exp \left(M \omega\left(\Psi_{0}\right)^{2} R_{0}^{2}\left(T_{i}+T_{e}\right)^{-1}\right\}\left[1+O\left(M \omega r\left(\Psi_{0}\right)^{2} R_{0}^{2}\left(T_{i}+T_{e}\right)^{-1}\right)\right],
$$

for $M \omega\left(\Psi_{0}\right)^{2} R_{0}^{2}\left(T_{i}+T_{e}\right)^{-1} \ll 1$, and

$$
\delta=1-O\left(\exp \left\{-\mathrm{M} \omega\left(\Psi_{0}\right)^{2} \mathrm{R}_{0}^{2}\left(\mathrm{~T}_{\mathrm{i}}+\mathrm{T}_{e}\right)^{-1}\right\}\right)
$$

for $M \omega\left(\Psi_{0}\right)^{2} R_{0}{ }^{2}\left(T_{i}+T_{e}\right)^{-1} \gg 1$. Therefore, the following simple connectiou formula is adequate for our regime of interest $M \omega\left(\Psi_{0}\right)^{2} R_{0}{ }^{2}\left(T_{i}+T_{e}\right)^{-1} \leq 1$,

$$
\delta=\left[1+(2 \varepsilon)^{-1 / 2} \exp \left(-M \omega\left(\Psi_{0}\right)^{2} R_{o}^{2}\left(T_{i}+T_{e}\right)^{-1}\right]\right]^{-1}
$$


The fraction of trapped electrons is easily shown to be the same as that of trapped ions and given by

$$
\delta=\left[1+(2 \varepsilon)^{-1 / 2} \exp \left(-\mathrm{M} \omega\left(\Psi_{0}\right)^{2} \mathrm{R}_{0}^{2}\left(\mathrm{~T}_{\mathrm{i}}+\mathrm{T}_{\mathrm{e}}\right)^{-1}\right)\right]^{-1}
$$

The $\theta$-dependence of the equilibrium density $n_{0}$ introduces a new term of the order of $M \omega\left(\Psi_{0}\right)^{2} L_{n} R_{0} T_{i}^{-1}$ smaller than the term we keep which originates from $\partial n_{0} \partial r$. Therefore, the conventional expression for the density response 14 is a good approximation.

For the dissipative trapped ion instability, $\omega_{t i}>\omega_{b i}>\omega_{* e}>v_{i, e f f}>\omega_{d i}$ and $\omega_{\text {te }}$ $>\omega_{b e}>v_{e, e f f}>\omega, \omega_{* e}>\omega_{\text {de }}$ are satisfied. Here, $\omega$ is the mode frequency in the rotating frame, $\omega_{* e}=C_{s} \rho_{s} k_{\theta} / L_{n}$ is the electron diamagnetic drift frequency, $\omega_{t e}$ and $\omega_{t i}$ are the electron and ion transit frequencie $\%$. The electron density response is given by

$$
\delta n_{\mathrm{e}} / n_{\mathrm{o}}=\left[1-\mathrm{i} 4\left(\varepsilon_{\mathrm{eff}} / \pi\right)^{1 / 2} \omega_{* \mathrm{e}}\left(1+3 \eta_{\mathrm{e}} / 2-\left(\omega / \omega_{* \mathrm{e}}\right)\right] /\left(\nu_{\mathrm{e}} / \varepsilon_{\mathrm{eff}}\right)\right] \mathrm{e} \delta \Phi / T_{\mathrm{e}},
$$

where $\delta \Phi$ is the perturbed electrostatic potential, and $\varepsilon_{\text {eff }}=\delta^{2} / 2 \geq \varepsilon$ characterizes the enhancement of the fraction of trapped particles. On the other hand, the ion density response is given by

$$
\delta n_{j} / n_{0}=-\left[\tau-\varepsilon_{\text {eff }} 1 / 2(\tau+\omega * e / \omega)+i v_{i i} \tau H / \varepsilon_{\text {eff }} \omega\right] e \delta \Phi / T_{e},
$$

where $\mathrm{H}=4\left(\varepsilon_{\mathrm{eff}} / \pi\right)^{1 / 2} \int_{\mathrm{d} v} \exp \left(-v^{2}\right) v^{-1}\left[1-\omega_{* \mathrm{i}}\left(1+\left(v^{2}-3 / 2\right) \eta_{\mathrm{i}}\right] / \omega\right]$ is a constant which depends on $\varepsilon_{\text {eff }}$ very weakly, ${ }^{31}$ and $\tau=T_{e} / T_{i}$. For both species, energy dependent simple Krook collisional operators $v_{\sigma, \text { eff }}=v_{\sigma} / \varepsilon_{\text {eff }}$ are used, ignoring the detailed shape of trapped-untrapped particle boundary in velocity space. Imposing quasineutrality on Eqs. (31) and (32), we get the following dispersion relation, 


$$
\begin{aligned}
& \operatorname{Re}(\omega)=\varepsilon_{\mathrm{eff}}^{1 / 2} \omega_{* \mathrm{e}} /(\tau+1), \\
& \operatorname{Im}(\omega)=4 \pi^{-1 / 2} \varepsilon_{\mathrm{eff}}^{2}\left(1+3 \eta_{\mathrm{e}} / 2\right) \omega_{*} \mathrm{e}^{2} /(1+\tau)^{2} v_{\mathrm{e}}-v_{\mathrm{ij}} \tau \mathrm{H} /(1+\tau) \varepsilon_{\mathrm{eff}} .
\end{aligned}
$$

We note that the toroidal rotation increases both the real frequency and the growth rate by enhancing the fraction of trapped particles. Damping due to ion-ion collision is reduced. From Eq. (34), we get the following criterion for the linear stabilization in a dense plasma,

$$
v_{\mathrm{e}} v_{\mathrm{ii}}>4 \pi^{-1 / 2} \varepsilon_{\mathrm{eff}}{ }^{3}\left(1+3 \eta_{\mathrm{e}} / 2\right) \omega_{*} \mathrm{e}^{2} /(1+\tau) \tau \mathrm{H}
$$

which is harder to achieve in the presence of toroidal rotation due to larger $\varepsilon_{\text {eff. }}$. We note that when collisional effects are analyzed with Lorentz collisional operators, the ion collisional damping term would scale as $v_{\mathrm{ii}}{ }^{1 / 2}$ instead of $v_{\mathrm{ii}}{ }^{32}$

Next, we consider the collisionless regime where $\omega_{\mathrm{ti}}>\omega_{\mathrm{hi}}>\omega, \omega_{* \mathrm{e}}>\omega_{\mathrm{di}}>v_{\mathrm{ii}} / \varepsilon_{\mathrm{eff}}$ and $\omega_{\mathrm{te}}>\omega_{\text {be }}>\omega, \omega_{* \mathrm{e}}>\omega_{\mathrm{de}}>v_{\mathrm{e}} / \varepsilon_{\text {eff }}$ are satisfied. ${ }^{14}$ In this case, the electron density response is given by

$$
\delta n_{e} / n_{0}=\left[1-\varepsilon_{e f f}{ }^{1 / 2}\left[1-\omega_{* e} / \omega+\left(3 \omega_{D e} / 2 \omega\right)\left\{1-\omega_{* e}\left(1+\eta_{e}\right) / \omega\right\}\right]\right] e \delta \Phi / T_{e} .
$$

For ions, we have

$$
\delta n_{i} / n_{0}=-\left[\tau-\varepsilon_{\text {eff }}^{1 / 2}\left[\tau+\omega_{* e} / \omega+\left(3 \tau \omega_{D_{i}} / 2 \omega\right)\left\{1-\omega_{* i}\left(1+\eta_{i}\right) / \omega\right\}\right]\right] e \delta \Phi / T_{e},
$$

where $1 /\left(\omega-\omega_{\mathrm{de}}\right) \equiv\left(1+\omega_{\mathrm{de}} / \omega\right) / \omega$ and $\varepsilon_{\mathrm{eff}} \ll 1$ has been used. $\omega_{\mathrm{Di}}$ and $\omega_{\mathrm{De}}$ are the precession drift frequencies evaluated at the thermal speeds and $G(x)$ has been approximated to be 1 , ignoring the pitch angle dependence. Quasineutrality condition yields the following 
almost purely growing instability,

$$
\gamma^{2}=\left(3 \varepsilon_{e f f}{ }^{1 / 2} / 2(1+\tau)\right)\left\{\left|\omega_{D_{i}}\right|\left(1+\eta_{i}\right)+\omega_{D e}\left(1+\eta_{e}\right)\right\} \omega_{* e}
$$

Therefore, the toroidal rotation has destabilizing influences through enhanced $\left|\omega_{\mathrm{Di}}\right|$, $\omega_{\mathrm{De}}$ and $\varepsilon_{\text {eff. }}$ We also expect destabilizing influences to the trapped ion modes propagating in the ion diamagnetic direction 33,34 which can resonantly interact with the trapped ion precession drift. In fact, a numerical capability for addressing the problem of the equilibrium electric field effects on tokamak microinstabilities in the context of one dimensional nonlocal analysis (the ballooning formalism) has already existed for many years. ${ }^{35}$ In Ref. 36 , this capability was utilized for a situation where the equilibrium poloidal electric field is the dominant modification. (However, in our case, the poloidal electric field effect is smaller than the centrifugal force effect on ions as shown in Eq. (11)). An equilibrium poloidal electric field in the form derived in Ref. 21, was used for a study of relatively shorter wavelength $(n=30)$ modes driven by the ion temperature gradient. No significant changes in the linear growth rate were observed when $\mathrm{e} \Phi_{1} / \Upsilon_{\mathrm{e}}$ was increased (decreased) up to (-)0.5 $\varepsilon \cos \Theta$. The local trends predicted in the present paper still need to be examined within the context of such nonlocal eigenmode calculations. 


\section{Discussions}

We have analyzed the effects of toroidal rotation on trapped particle orbits and the local stability properties of trapped ion modes. Toroidal rotation induced radial electric field enhances the magnetic trapping of ions, meanwhile the centrifugal force induced poloidal electric field enhances the magnetic trapping of electrons. This increases the fraction of trapped particles and their precession frequencies. Consequently, the local growth rate of trapped ion instability increases in both dissipative and collisionless regimes. The banana radial width of a trapped ion in the presence of rotation shear is derived, and a discussion is given to elucidate the relation between two seemingly different previous results. 16,17

Also from our results on trapped electron orbit analysis, we can expect the following toroidal rotation effects on the trapped electron instabilities in the context of local theory. First, in the linear regime, there exists a destabilizing influence on the trapped electron modes in both dissipative and collisionless regime through the enhanced fraction of trapped electrons. Further destabilization is expected for dissipative trapped electron mode from the decrease of $v_{e, e f f}$, and for collisionless trapped electron mode from the increase of $\omega_{\mathrm{de}}$. Second, in the nonlinear regime, faster trapped electron precession drift can enhance the trapped electron Comnton scattering 37,8 of the collisionless trapped electron modes resulting in a stronger $f(d$ spectral transfer of fluctuation energy. Finally, the afore-mentioned modifications of various frequencies related to particle orbits can affect the strengths of excitation or damping mechanisms of instabilities other than the trapped particle modes. These possibilities are currently under investigation and will be published in the near future. 


\section{Acknowledgments}

The author would like to thank Y. B. Kim and P. H. Diamond for initiating his interest in this problem and for numerous stimulating discussions. He has benefited from conversations with S. C. Cowley on the orbit problerns. He is also grateful to W. M. Tang and G. Rewoldt for useful conversations on the trapped particle instabilities.

This work. was supported by U.S. Department of Energy Contract No. DE-ACO2-76-CHO-3073. 


\section{References}

1 E. Mazzucato, Phys. Rev. Lett 48, 1828 (1982).

2 N. Bretz, Rev. Sci. Instrum. 61, 3031 (1990).

3 E. Mazzucato and R. Nazikian, Plasma Phys. Controlled Fusion 33. 261 (1991).

4 R. J. Fonck, P. A. Duperrex, and S. F. Paul, Rev. Sci. Instrum. 61, 3487 (1990).

5 P. L. Similon and P. H. Diamond, Phys. Fluids 27, 916 (1984).

6 F. Y. Gang, P. H. Diamond, and M. N. Rosenbluth, Phys. Fluids B 3, 68 (1991).

7 T. S. Hahm and W. M. Tang, Phys. Fluids B 3, 989 (1991).

8 T. S. Hahm, Phys. Fluids B 3, 1445 (1991).

9 S. Scott, P. Diamond, R. Fonck, R. Goldston, R. Howell, K. Jaenig, G. Schilling, E. Synakowski, M. Zamstorff, C. Bush, E. Fredrickson, K. Hill, A. Janos, D. Mansfield, D. Owens, H. Park, G. Pautasso, A. Ramsey, J. Schivell, G. Tait, W. Tang, and G. Taylor, Phys. Rev. Lett. 64, 531 (1990).

10 P. H. Diamond and H. Biglari, Phys. Rev. Lett 65, 2865 (1990).

11 B. A. Carreras, K. Sidikman, P. H. Diarnond, P. W. Terry, and L. Garcia, Submitted to Phys. Fluids B (1992).

12 T. S. Hahm, Princeton University, Plasma Physics Laboratory Report PPPL-2830 Submitted to Phys. Fluids B (1992).

13 F. L.Waelbrock, T. M. Artonsen, Jr., P. N. Guzdar, and A. B. Hassam, Submitted to Phys. Fluids B (1992).

14 B. B. Kadomtsev and O. P. Pogutse, Soviet Physics JETP 24, 1172 (1967).

15 G. Rewoldt and W. M. Tang, Phys. Fluids B 2, 318 (1990), W. M. Tang and G. Rewoldt, Paper 1C23, in Proceedings of Sherwood International Theory Conference (Santa Fe, New Mexico, 1992).

16 R. D. Hazeltine, Phys. Fluids B 1, 2031 (1989). 
17 Y. B. Kim, Bull. Amer. Phys. Soc. 36, 2424 (1991).

18 F. L. Hinton and S. K. Wong, Phys. Fluids 28, 3082 (1985).

19 J. W. Connor, S. C. Cowley, R. J. Hastie and L. R. Pan, Plasma Phys. Controlled Fusion 29, 919 (1987).

20 T. S. Hahm, in Proceedings of International Sherwood Fusion Theory Conference, Paper 2C 6 (Santa Fe, New Mexico, 1992).

21 C. S. Chang and R. Harvey, Nucl. Fusion 23, 935 (1983).

22 S.-I. Itoh and K. Itoh, Nucl. Fusion 29, 1031 (1989).

23 K. L. Wong and C. Z. Cheng, Phys. Fluids B 1, 545 (1989).

24 H. L. Berk and A. A. Galeev, Phys. Fluids 10, 441 (1967).

25 H. P. Furth and M. N. Rosenbluth, in Plasma Phys. Controlled Nuclear Fusion Research (IAEA, Vienna, 1969) Vol. 1, p.821.

26 S. C. Cowley and F. W. Perkins, private communications (1989).

27 R. J. Taylor, M. L. Brown, B. D. Fried, H. Grote, J. R. Liberati, G. J. Morales, P. Pribyl, D. Darrow, and M. Ono, Phys. Rev. Lett. 63, 2365 (1989).

28 R. J. Groebner, K. H. Burreli, and R. P. Seraydian, Phys. Rev. Lett. 64, 3015 (1990).

29 Ch. P. Ritz, R. D. Bengston, S. J. Levinson, and E. J. Powers, Phys. Fluids 27, 2956 (1984).

30 K. Ida, S. Hidekuma, Y. Miura, T. Fujita, M. Mori, K. Hoshino, N. Suzuki, T. Yamauchi, and JFT-2M Group, Phys. Rev. Lett. 65, 1364 (1990).

31 G. Rewoldt, private communications (1982).

32 M. N. Rosenbluth, D. W. Ross, and Kostamarov, Nucl. Fusion 12, 3 (1972).

33 W. M. Tang, J. G. Adam, and D. Ross, Phys. Fluids 20, 430 (1977).

34 M. Tagger, G. Laval, and R. Pellat, Nucl. Fusion 17, 109 (1977).

35 G. Rewoldt, W. M. Tang, and M. S. Chance, Phys. Fluids 25, 480 (1982).

36 W. M. Tang and G. Rewoldt, Bull. Amer. Phys. Soc. 27, 1105 (1982). 
37 L. Chen, R. L. Berger, J. G. Lominadze, M. N. Rosenbluth, P. H. Rutherford, Phys. Rev. Lett. 39, 754 (1977). 
Dr. F. Peoboni, Univ. of Wollongong. AUSTRALIA

Prot. M.H. Brennen, Univ. of Sydnoy, AUSTRALIA

Plasma Racoarch Lob., Austrition Nat Univ., AUSTRALIA

Prol. I.R. Jones, Finders Univ, AUSTRALIA

Prol. F. Cep, Inst for Theoredical Phycics, AUSTRIA

Prot. M. Hoindier, Inscitue tor Theoreteche Phyeik, AUSTAIA

Prof. M. Cooseons, Autronomisch InetituUt, BELGIUM

Ecole Royad Nituin, Lob. do Phy. Plaemas, BELGIUM

Commizaion-Europaen, DG. XII-Fusion Prog., BELGIUM

Prof. R. Boudiaus, Rakeuninuthin Gent, BELGIUM

Dr. P.H. Saknoma, Instuto Fivica, BRAZIL

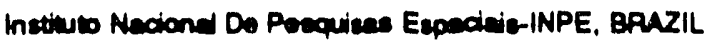

Documents Orioe, Atomic Enorgy of Canada Ld., CANADA

Dr. M.P. Bectrynski, MPB Tectnologios, Inc., CANADA

Dr. H.M. Skaregand, Univ. of Saskakhowen, CANADA

Prof. J. Toictimam, Univ. of Montred, CANADA

Prot. S.R. Sroconivasen, Univ. of Culgary, CANADA

Prof. T.W. Johneton, INRS-Energis, CANADA

Dr. R. Botton, Contre cantadion de tusion magnetique, CANADA

Dr. C.R. James., Univ. of Nborta, CANADA

Dr. P. Lukte, Komenatho Univernzita, CZECHOSLOVAKIA

The Librarian, Cuham Leborabry, Ei 3LAND

Librery, R61, Ruthertord Applotion Laboratory, ENGLAND

Mrt. S.A. Hutchinion, JET Liorary, ENGLAND

Dr. S.C. Sheme, Univ. of South Pecific, FIJI ISLANDS

P. Mahonen, Univ. of Helsinix, FINLAND

Prof. M.N. Buacenc, Ecoiv Poty cotnique, FRANCE

C. Moutuat, Lob. do Phycique des Milioux lonises, FRANCE

J. Radot, CEMCAOARACHE - Bat 506, FRANCE

Prot. E. Economou, Univ. of Crm, GPEECE

Ms. C. Rinnd, Univ, of loamine, GREECE

Dr. T. Mull, Acadermy Bibiographic Ser., HONG KONG

Proprinit UJbrery, Hungarian Acadomy of Sa., HUNGAPY

Dr. B. Descupta, Saha inst of Nucber Phycics, INDIA

Dr. P. Kaw, Inst. for Plesma Rescarch, INDIA

Dr. P. Rocenew, lereed inst of Technology, ISRAEL

Litrarien, Intornationd Comber for Theo Physics, ITALY

Niss C. Do Pab, Associazions EURATOMENEA, ITALY

Dr. G. Groseo, Istiuto di Fieica ded Praema, ITALY

Prol. G. Rostenoni, lectaro Gas lonizzan Dol Cnr, ITALY

Dr. H. Yemwo, Tochiba Res a Dowl Conier, JAPAN
Prot. I. Kawakemi, Hroshima Univ., JAPAN

Prof. K. Nishikeme, Hroehima Univ., JAPAN

Drector, depen Atomic Enorgy Rocoarch inst, JAPAN

Prot. S. Iroh, Kyushu Univ., JAPAN

Peseurct Into. Cr., Nationd Instit. for Fusion Science, JAPAN

Prot. S. lieneka, Kyoso Univ., JAPAN

Library, Kyilo Univ., JAPAN

Prof. N. Inoun, Univ. of Tohyo, JAPAN

Socretary, Plama Section, Electronochnical Lab., JAPAN

5. Mori, Technicad Advisor, MAEP, MAPAN

Dr. O. Miend, Kumenoto inst. of Tectnology. JAPAN

J. Hyeon-Sook, Korea Alomic Enorgy Recearch Inst, KOREA

D.I. Chal, The Korea Adv. Inst. of Sa. \& Tech., KOREA

Prot. 8.S. Liby, Univ. of Waikat, NEW ZEALAND

Inat of Phyrics, Chinece Acad Sa PEOPLE'S REP. OF CHINA

Librery, Inat of Plasma Physios, PEOPLE'S REP. OF CHINA

Tainghua Univ. Library, PEOPLE'S REPUBLIC OF CHINA

Z. Li, S.W. Inat Phyeics, PEOPLE'S REPUBUC OF CHINA

Prof. J.A.C. Cebred, Instituto Superioc Tecnico, PORTUGAL

Dr. O. Potrus, Al I CUZA Univ., ROMANIA

Dr. J. do Vilion, Fusion Studias, AEC, S. AFRICA

Prot. M.A. Helberg, Univ. of Natal, S. AFRICA

Prot. D.E. Kim, Pohang Inet. of Sai. I Tech., SO. KOREA

Prot. C.I.E.M.A.T, Fusion Division Library, SPAIN

Dr. L Sionflo, Univ. Of UMEA, SWEDEN

Librery, Royd inat. of Tectnology, SWEDEN

Prot. H. Whatemcon, Chelmore Univ. of Tech., SWEDEN

Centre Phys. Des Plaemas, Ecole Potytech, SWITZERLAND

Bibliotheak, inst. Voor Plasma-Fysica, THE NETHERLANDS

Aset Prot. Dr. S. Cakir, Midolo East Tech. Unir., TURKEY

Dr. V.A. Gulkikh, Sci. Ras. Inst Eloctrophye.I Apparatus, USSR

Dr. D.D. Ayurov, Siberian Brench of Acodomy of Sa., USSA

Or. G.A. Eisoev, I.V. Kurchatov Inst, USSA

Librevien, Tho Ukr.SSR Acadomy of Scionces, USSR

Dr. LM. Kowizhmykh, Inst. of Gonerd Physics, USSR

Kemtorectungeandege $\mathrm{GmbH}$, Zentrabibliothex, W. GEHMANY

Bibliothok, inst. For Phasmatorectung. W. GERMANY

Prof. K. Schinder, Ruhr-Univervitt Bochum, W. GERMANY

Dr. F. Weoner, (ASDEX), Max-Plenck-Inetitue, W. GEAMANY

Lbrevion, Max-Plenck-Institux, W. GERMANY

Prol. R.K. Janev, Inat of Phyaics, RugosLaVIA 

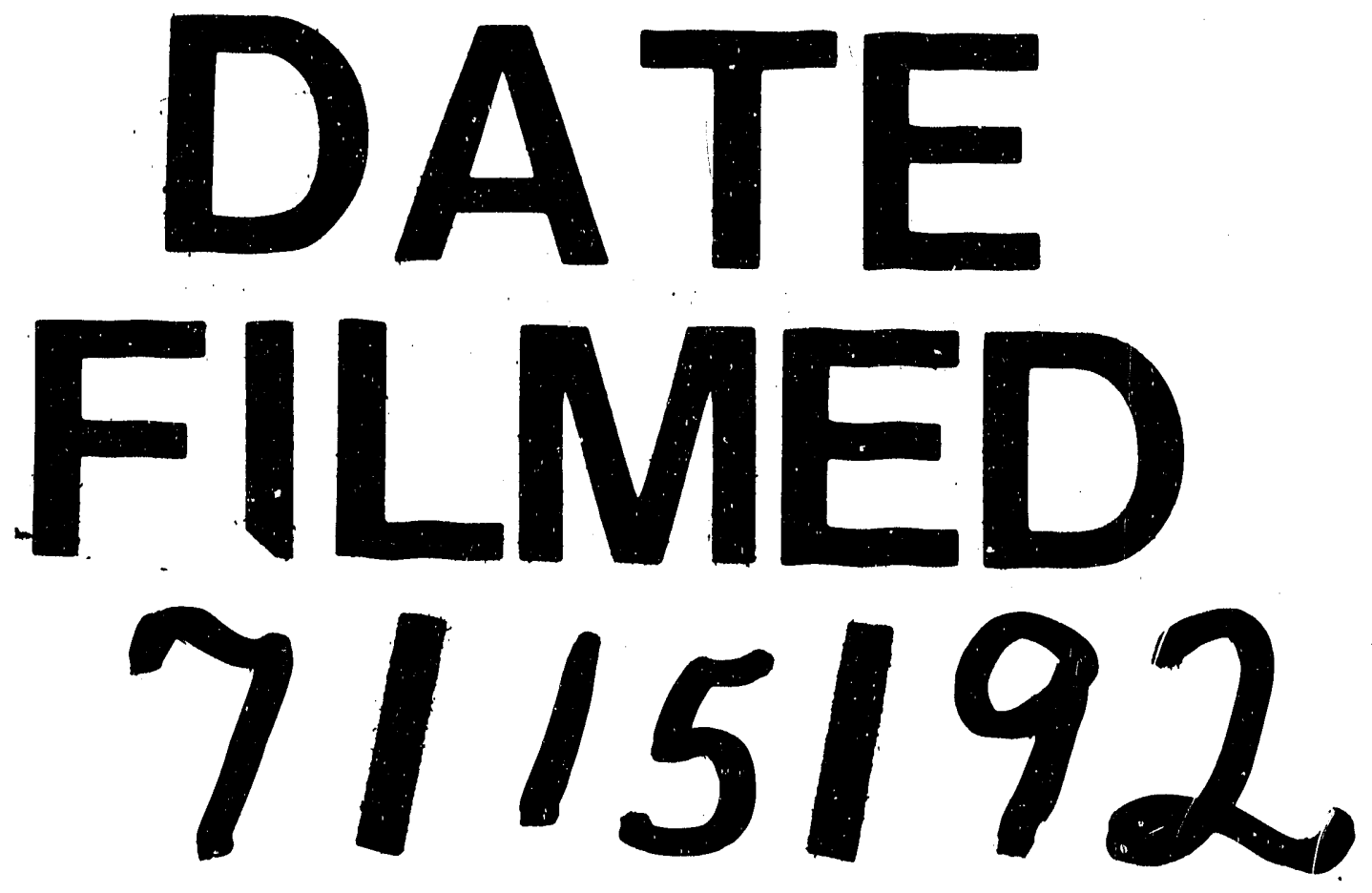
